\title{
A Study of Two-Handed Scrolling and Selection on Standard Notebook Computers
}

\author{
Dominik Bial \\ Pervasive Computing \\ University of Duisburg-Essen, Germany \\ dominik.bial@uni-due.de
}

\author{
Florian Block \\ Computing Department \\ Lancaster University, UK \\ block@comp.lancs.ac.uk
}

\author{
Hans Gellersen \\ Computing Department \\ Lancaster University, UK \\ hwg@comp.lancs.ac.uk
}

\begin{abstract}
Although two-handed input can improve both efficiency and quality of user interaction, it is not commonly adopted as it requires additional input devices. In this paper we propose two-handed interaction on standard hardware - notebooks with external mouse - and for a common task - 2D scrolling. We introduce four techniques that leverage the built-in touchpad as a dedicated scrolling device for the non-dominant hand, for scenarios in which the mouse is used in parallel for object selection and manipulation tasks. The techniques implement relative scrolling, flicking, absolute positioning and token-based input on the touchpad. We present an empirical evaluation of these techniques in a task that simulates activities such as retouching of photos, or interaction with maps, in which users often switch between mouse interaction and scrolling. The results show initially best performance with relative scrolling as a familiar mapping, but strong learning effects for all techniques. Users had difficulty with absolute mapping of touchpad input due to a tendency to clutching and finger repositioning, but we observed that these problems are compensated when a token is used as absolute input device.
\end{abstract}

Keywords: Two-Handed Interaction, Scrolling, Touchpad, Notebook.

\section{INTRODUCTION}

People naturally use both hands when performing many common tasks, such as driving, cooking or playing an instrument. A large body of research in $\mathrm{HCl}$ has investigated how two-handed interaction skills can also be applied to interaction with computers. It has been shown that two-handed interaction can provide a variety of benefits, such as increased efficiency, parallelism of input, and improved expressiveness and quality of user interaction $[4,10$, $12,6,13,11]$.

In practice, two-handed interaction is commonly only found in niche application areas, such as CAD or video editing. In these contexts, workstations are extended with specialized input hardware for the non-dominant hand, such as a 3D mouse [1]. However, there is still no wide-spread support for two-handed interaction for the majority of common workflows, such as photo-editing, navigating maps or browsing the web. In spite of the known benefits of two-handed interaction, most users carry out the diverse interactive tasks involved in such workflows using a single pointing device.

In this paper, we propose two-handed interaction on notebooks that are used with an external mouse ("N+M"), a configuration in which two continuous input devices are readily available "out-of-the-box". All notebooks have a built-in pointing device, most commonly a touchpad, but many users prefer to attach an external mouse as pointing device, in particular when they use their notebook in a regular stationary setting such as their office or their home. In an N+M configuration, the built-in touchpad is presently rendered completely redundant. We propose to tap into this unused potential, and to use the touchpad as a secondary input device for scrolling with the non-dominant hand, while control over the mouse pointer remains with the dominant hand. Scrolling is a very common task that can be found in a wide variety of applications, such as editing of zoomed-in images, navigation of maps or scrolling through web pages. The aim is to provide the benefits of two-handed interaction for a broad user base on a platform that is already widely available.

The contribution of this paper is two-fold. The first contribution are four different techniques that leverage the touch-pad as 2D navigation device, and enable two-handed interaction in a standard configuration of notebook with mouse. Two of the introduced techniques are based on a relative mapping, one mapping relative finger movement on the touch-pad to relative scrolling, and the other using flick gestures for relative scrolling in the indicated direction. The other two techniques are based on an absolute mapping of a position selected on the touchpad to a position in the target space, one using finger position and the other position of a token 
placed on the touchpad. Consider also figure 2 for more details. The second contribution is an empirical evaluation in which we compare the four techniques in a realistic task. In this task, users use their nondominant hand on the touchpad to scroll across a zoomed-in image, while using the mouse with the dominant hand for selection of a small target in the image. We report on both quantitative and qualitative findings and discuss the results and implication for the suitability of the touchpad as dedicated 2D scrolling device for $\mathrm{N}+\mathrm{M}$ configurations.

\section{RELATED WORK}

According to sales trends, notebooks are becoming more widely adopted for desktop interaction than traditional PCs, but the input configurations in which they are used have received only limited research attention. Prior studies include surveys of different user groups with respect to ergonomics of notebook use $[9,18]$, comparative evaluation of built-in pointing devices [7, 17] and ethnographies of notebook usage in the home [19]. It has been observed before that notebooks are often used with an external mouse as pointing device [7], but this work is first to propose that the built-in pointing device can then be used for complementary input with the non-dominant hand.

Prior work has shown that capacitive touch-sensing surfaces can be used for other forms of input that are distinct from mouse-style pointing [5], including gestural input $[4,15]$, and token-based input using pucks [10]. These techniques have not previously been considered for use on notebook touchpads. However, an example of touchpad use beyond pointing has been demonstrated in ThumbSense, where touchpad input is used as a key modifier for emulation of mouse buttons on the keyboard [16].

The proposed «activation» of the notebook touchpad complementary to an external mouse facilitates interaction with two continuous input devices, with the potential to leverage a large body of research on two-handed input [6]. The configuration is inherently asymmetric, first in that the two devices have different and distinctive properties $[5,10]$, and secondly in that users have an a priori preference for the mouse as primary pointing device. Guiard's Kinematic Chain model is therefore of key relevance to our work as it provides theoretical insight into principles governing asymmetric two-handed interaction [8]. The model describes a distinct role for the non-dominant hand, in setting the frame of reference in which the dominant hand operates («Right-to-left Spatial Reference in Manual Motion»). It further proposes that the nondominant hand performs less fine-grained movement («Left-Right Contrast in the Spatial Temporal Scale of Motion»). This corresponds with experimentally observed left-right-hand input performance, finding that the non-dominant hand is well suited for tasks that do not require precise action, such as scrolling [12].

There are a number of two-handed techniques reported in the literature in which the non-dominant hand perform roles complementary to the dominant hand, including scrolling and navigation $[6,13,1$, 20], moving toolglasses [11, 13, 14] and performing gestures [4]. In all of these cases, the dominant hand is used for fine-grained positioning, which naturally maps to mouse operation in an $\mathrm{N}+\mathrm{M}$ scenario, while the non-dominant hand performs actions that, in principle, can be realised on notebook touchpads.

The specific combination of touchpad and mouse has been previously investigated, however with a touchpad as external device [10], rather than integrated to be close to hand from the keyboard. It is important to note conceptual differences between mouse and touchpad in such a configuration, and to understand that the devices operate in different kinaesthetic reference frames. However general techniques such as context setting and moving toolglasses can be adapted to work with relative input from both devices, or alternatively with the touchpad as absolute and the mouse as relative input device. Research also suggests that separation of kinaesthetic reference frames in twohanded interaction can be compensated with visual feedback [3].

\section{SCENARIO}

Scrolling is a generic task that is part of many common workflows, usually performed in one or two dimensions. ID scrolling applies for instance to a word processor, for scrolling up and down in documents. 2D scrolling is common for instance for panning across zoomed images and navigating maps that are larger than the display space. In this work, we focus on 2D scrolling or panning. This is motivated by the nature of touchpads as $2 \mathrm{D}$ input device. Moreover, 2D scrolling techniques can be projected for ID scrolling, by simply disregarding one of the two dimensions. This means that the techniques we introduce as well as some of the results from our empirically evaluation can be transferred to ID scrolling.

In many common applications (such as Photoshop, GIMP or Corel Draw), 2D scrolling is performed with the mouse via a navigator widget (cf. Fig. 1). The navigator widget displays a thumbnail of the complete content that the user is navigating, as 


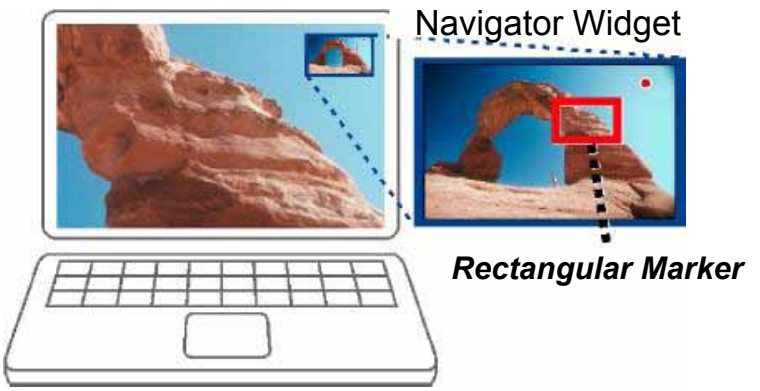

Figure 1: A navigator widget is a common tool for $2 D$ scrolling. It shows a thumbnail of the overall content and a rectangular marker that indicates the portion of the image that is displayed in the main view. The rectangular marker can be moved by clicking on the thumbnail or by

drag\&drop to the effect of scrolling the main view.

well as some form of rectangular marker that indicates which portion of the overall content is currently displayed in the main view. Users can then either jump to a specific position by clicking on the respective position of the thumbnail, or drag\&drop the rectangular marker across the thumbnail, which continuously scrolls the content across the main view.

There are many workflows in which scrolling is a significant part of the overall interaction. For our study and for illustration purposes, we use the process of retouching an image. In such a scenario, the user frequently switches between scrolling the currently zoomed view via the navigator and manipulating details in the zoomed image. In most state of the art operating systems, both activities scrolling and manipulating the image - are executed via the mouse. This requires the user to frequently switch the mouse cursor between the navigator widget, and the actual area of the zoomed picture which has to be retouched. The opportunities in using the touchpad as a dedicated scrolling device are to reduce mouse movement, to provide the user with more direct access to scrolling, and to enable novel ways of scrolling that cannot be accomplished through conventional pointer input.

The described workflow is not limited to image editing but is common in many other applications. Examples include navigation of a map while adjusting the way points of a route (Google Maps), manipulation of splines of a vector graphic that extends the display space (Corel Draw), and map navigation in a strategy game while selecting and commanding units (Warcraft III). Consequently, the following techniques, as well as the results of the empirical evaluation, can be widely applicable for interaction with notebook computers.

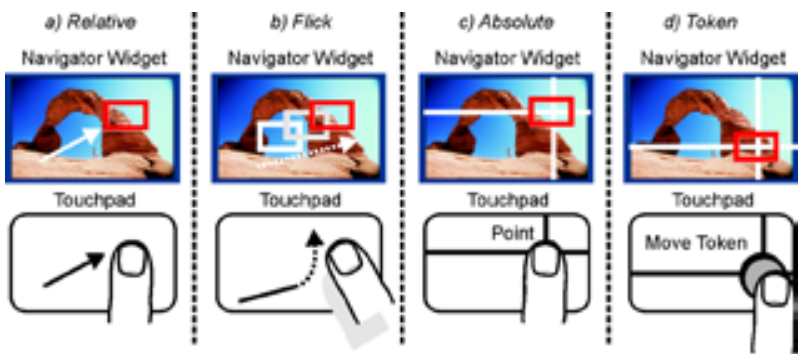

Figure 2: Scrolling techniques for the touchpad.

\section{TOUCHPAD SCROLLING TECHNIQUES}

On the lowest level, a touchpad provides a stream of raw input values, including movement and proximity of the finger, and absolute position of the finger on the touchpad. Usually, these values are mapped in a way that moves the mouse pointer very similar to a mouse. In $\mathrm{N}+\mathrm{M}$ configurations, the touchpad can be decoupled from conventional pointer input, and the provided values can instead be mapped onto navigation tasks. We introduce four techniques that use low-level touchpad sensor data for such an alternative mapping. The low-level data on which these techniques are based are supported by most state of the art touchpads. The techniques are:

- Relative: This technique is similar to mouse pointer control with a touchpad in as far as using relative movement on the touchpad as input, however this input is mapped to relative scrolling in two dimensions (see Fig. $2 a$ ). When the user moves the finger on the touchpad in a specific direction the content in the main view is scrolled correspondingly. In our scenario, moving the finger to the right would cause the rectangular marker in the navigator widget to move to the right as well, effectively moving the content to the left and revealing more content to the right of the current view. As the touchpad provides limited space for relative movement, users can lift their finger up, move it back and repeat relative movement in the same direction. This technique is called clutching, and is familiar from touchpad use for mouse pointer control. It allows users to move into any direction, theoretically infinitely.

- Flicking: This scrolling technique is also based on relative movement but allows to apply momentum to scrolling, by flicking the finger off the touchpad's surface into a target direction. The underlying metaphor is to consider the rectangular marker on the navigator as an object that once put into motion can slide across the thumbnail view, 
and thus continuously scroll the main view (cf. Fig. 2b). Virtual friction will reduce the momentum of the current view and make it stop eventually, but users can also halt the movement by bringing the finger down on the touchpad. Flicking has the advantage that the user can scroll over large distances with little movement of the fingers, and without need for clutching.

- Absolute: Contrary to the above techniques, Absolute is based on absolute finger position on the touchpad as input. The touchpad area represents the controlled virtual space, in our scenario the edited image in its full extent. Pointing to a specific position on the touch-pad moves the rectangular marker to the corresponding absolute position in the thumbnail representation, and scrolls the image accordingly (cf. Fig. 2c). The finger can also move across the touchpad, however, unlike Relative, scrolling is updated according to the absolute position on the touchpad, instead of relative movement.

- Token: Like the previous technique, Token uses an absolute mapping, however instead of direct finger input, a token is used on touchpad for selection of an absolute position. The technique was inspired by use of pucks or similar devices on input tablets [10]. Notebook touch-pads are not designed for operation with a token, but in practice token-based input can be achieved by adding a metal token, for example a coin, to the touchpad. A metal token that is touched on the touchpad amplifies the capacitive footprint of the finger and can be distinguished from direct finger touches. The token acts as both indicator as well as handle for the current view position in the overall image space. The input provided by the technique is exactly the same as with Absolute but the use of a token encourages a different style of interaction, namely to adjust the position of a physical token, rather than to point directly to a specific location with one's finger.

All four techniques employ principles that have been demonstrated in other contexts. Flicking, for example, is familiar from interfaces such as the iPhone, and both absolute and token-based input are known from input tablets. The provision of these techniques on standard notebook touchpads however is novel, and opens up new interaction opportunities that can be readily adopted in $\mathrm{N}+\mathrm{M}$ configurations, i.e. when notebooks are used with an external mouse.

\section{EMPIRICAL EVALUATION}

The techniques we have introduced in the previous section constitute different approaches to mapping the touchpad as a dedicated device for scrolling. In the following we describe an empirical evaluation, in which we assess quantitative and qualitative aspects of each technique.

\subsection{Design \& Participants}

The study used a $5 \times 3$ factorial design with two factors Input Technique and Block. Scrolling Technique included the four touchpad scrolling techniques Relative, Flick, Absolute and Token, and conventional $2 \mathrm{D}$ scrolling with the mouse and navigator widget as the control condition Mouse. The factor Block captures the learning effect with each technique, and has three levels (Block 1, Block 2 and Block 3) which represent the first, second and last set of trials of the task (10 trials per set). However, the levels of blocks were created after the test. During the experiment, users executed all thirty trials consecutively without interruption.

15 participants were paid to participate in the study ( 9 female, 5 male). The age ranged from 19 to 50 years, with an average of 28.1 years. Two participants were left-handed but still preferred to use the mouse with the right hand. 13 of the participants owned a laptop, in which a touchpad is integrated. $53.8 \%$ of the laptop owners reported to prefer an external mouse over the built-in touchpad.

The dependent variable was task completion time. Additionally, all mouse and touchpad movements were recorded for further analysis, and each experiment was video taped. All post-hoc tests were corrected using Bonferroni.

\subsection{Task}

The study task was derived from image retouching, as described in our scenario. In each trial, a user first scrolls the view to reveal a specific portion of the image and then performs a detailed manipulation on the current view. Within one session, this process repeats until all interactions are performed. Figure 3 shows the tasks each participant had to perform. The screen showed a simplified image editor, providing a main view and the navigator widget. The checkered pattern represents a generic image, on which the user operates. In each trial, the navigator will first show a yellow area, to which the user has to navigate. Once the yellow area comes into the main view, the user might have to perform further scrolling across the yellow area in order to find a red dot, which simulates the actual target of interaction. 


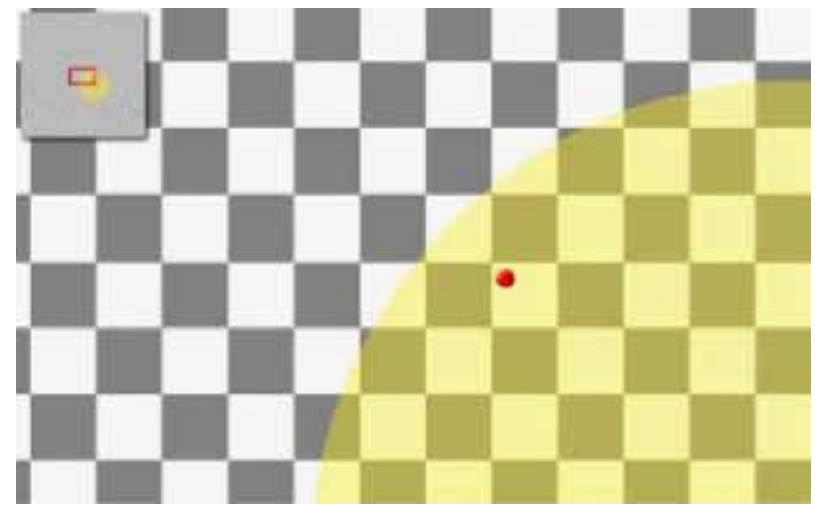

Figure 3: Study task

This two-stage approach is designed to reflect realistic scrolling behaviour. First, the user scrolls to the rough area she wants to retouch (e.g., the face of a person in the picture), using the navigator. Due to the limited detail provided by the thumbnail view, it is usually difficult to identify detail of interest (e.g., impurities of the skin that are to be touched up) to directly zoom into. The user thus typically needs to scroll the target area to bring the desired detail into focus in the main view. In a realistic task, the eventual interaction will involve selection of image detail and use of filters. For the purpose of experimental evaluation we simulate the detail of interest by a red dot, and limit the mouse task to selection by a click on the dot. Clicks are sufficient for our task, in that they introduce the necessity to accurately move the mouse pointer to a target detail in the image, and capture the navigational aspects of the overall workflow. After a user has clicked on the red target, the next target area is revealed on the navigator, and the process is repeated. The chosen sequence of target areas and red dot was chosen in a way that a new target would never lie in the currently zoomed view. Consequently, for each trial, navigation was necessary.

In our control condition Mouse, participants had to navigate in the conventional way, namely by using the mouse to manipulate the rectangular marker. Participants could either drag and drop the rectangular viewfinder across the thumbnail representation, or simply click on any point on the thumbnail, causing the marker to jump directly to that location. In the other four scrolling techniques, the touchpad acted as a dedicated device for manipulating the rectangular marker. All touchpad techniques were used as described in the previous section and illustrated in Figure 2.

\subsection{Procedure}

Each participant was first given an introduction to the study and the task they were about to perform. All participants then carried out the described task with each of the five levels of Input Technique.
The order of the techniques was balanced across all 15 participants using latin-square. For each of the five conditions, participants had to execute thirty consecutive trials as described. The position of the target areas (yellow circles) as well as the actual target point within the area (red dots) were pseudo-randomized in a way that each condition had a different set of target areas and points, while ensuring that the overall navigation distance was the same for each condition.

After each of the conditions participants were given a questionnaire to rate the scrolling technique and provide qualitative feedback. After the last condition, participants were also asked for a comparative rating, and to provide general feedback. The whole study took about 50 minutes for each participant.

\subsection{Apparatus}

The study was run on a standard notebook (FSC Amilo Si 3655) with a 13.3 inch display and $1280 \times 800$ screen resolution, using its built-in Synaptics touchpad and a standard laser mouse. The touchpad in our particular model measured 65 $x 40 \mathrm{~mm}$. For the navigation task, an image with a size of $6000 \times 6000$ pixels was used. All interaction techniques were implemented using the Synaptics API, which allows the touchpad to be decoupled from pointer input, and which provides access to the raw values of the touch-pad, such as absolute position of the finger, movement, and capacitive strength of the contact point [2].

\subsection{Results}

\subsubsection{Task-Completion Time}

Effects of Input Technique. An ANOVA showed a significant main effect oí Scrolling Technique (F4, $56=7.168, p<0.001)$. Relative performed fastest (mean 101.1s, s.d. 19.1s) followed by Mouse (mean 104.1s, s.d. 26.9s), Flick is (mean 111.8s, s.d. 17.2s), Token (mean 126.3s, s.d. 22.4s) and Absolute (mean 133.2s, s.d. 35.5s). However, the post-hoc test shows significant differences only between Mouse and Token ( $p=0.036)$, Token and Relative $(p=0.004)$ as well as Relative and Absolute $(p=0.038)$.

Effect of Block. There is a significant main effect for Block (F2, $28=7.168, p<0.001)$. Post-hoc test showed that participants could significantly improve their performance from Block 1 (mean 41.6s, s.d. $17.8 \mathrm{~s})$ to Block 2 (mean 37.9s, s.d. 17.4s) $(p=0.044)$. However, there was no significant improvement from Block 2 to Block 3 (35.8s, s.d. 13.3s) 


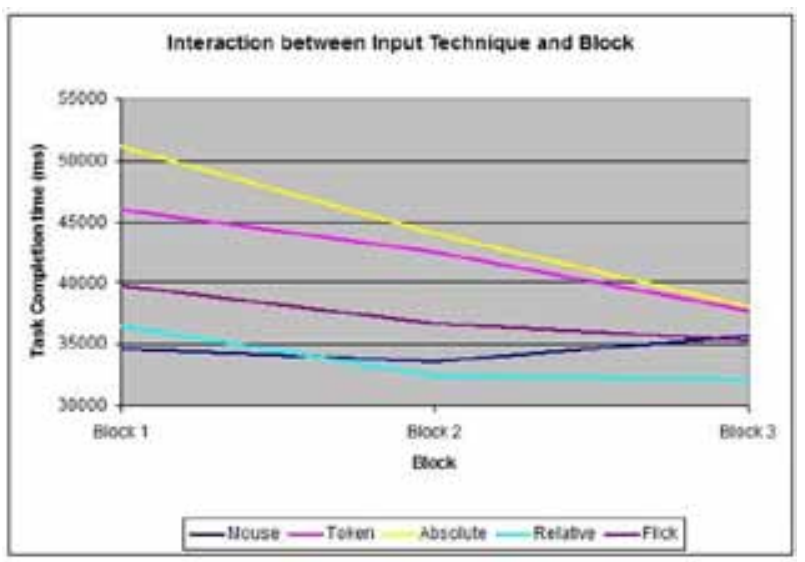

Figure 4: Learning Effect

Learning Effect There was a significant interaction between Scrolling Technique and Block (F8, $112=$ $2.409, p<0.019$ ). Figure 4 shows the learning curves for each technique across the three blocks. Mouse, the control condition, showed no classical learning behaviour, which was expected due to the fact that users are trained in this form of scrolling. In contrast, both absolute techniques, Absolute and Token show a steep learning curve across all three block. Despite the worst initial performance, the learning curves indicate that with both absolute scrolling techniques users had not reached their full performance within the short duration of the experiment. Both relative techniques also showed a learning curve, which is more pronounced between the first and second block.

\subsubsection{Overshoots}

Based on the recorded activity log, we also determined the amount of overshoots of each scrolling technique. An overshoot was counted, when first the target (red dot) was made visible in the main view, however, the user continued to scroll, causing the target to leave the main view again. The number of overshoots gives us an idea of how precisely users were able to navigate to the target with each of the techniques.

A separate repeated measurement factorial design was used, with one factor Input Technique (identical to the orignal study design) and the dependent variable Overshoots. An ANOVA shows a significant main effect for Input Technique (F4, $56=41.668$, $p$ $<0.001$ ). Relative produced least overshoots (mean 6.3, s.d. 2.6), followed by Mouse (mean7.6, s.d. 4.8), Flick (mean 10.2, s.d. 4.5) and Absolute (mean 12.1 , s.d. 4.5). Token caused the highest amounts of overshoots (mean 31.1, s.d. 10.6). The post-hoc test shows that these differences are significant between Relative and Flick $(\mathrm{p}=0.035)$, and Absolute and Relative $(p=0.003)$. Additionally, Token produced significantly more overshoots than any other technique $(p<0.001)$.

\subsubsection{Optimum Distance Ratio}

While the sequence of target locations was different for each condition, the overall distance between all targets was constant $(77,972$ pixels). This distance captures the minimum possible distance that had to be scrolled across, since it is the sum of the shortest distances between each consecutive pair of target locations in the sequence. Practically, participants were unlikely to achieve the minimum distance, since participants often deviate from the direct line between previous and current target and additionally have to search the target area for the actual target. However, the ratio between minimum distance and actual distance would provide us with data about how direct users could navigate to the targets with each technique.

The average actual distances were calculated for each participant and condition. The Optimum Distance Ratio is the actual distance navigated divided by the minimum distance. Based on the Optimum Distance Ratio as dependent variable, another repeated measurement factorial design was used, with the factor Scrolling Technique (levels identical to previous designs). An ANOVA shows a significant main effect for Scrolling Technique ( $F$ 4, 56 $=20.593, p<0.001)$. Relative (mean 1.28, s.d. 0.14) was the most direct technique, followed by Mouse (mean 1.39, s.d. 0.14), Flick (mean 1.43, s.d. 0.12), Token (mean 2.04, s.d. 0.24) and Absolute (mean 2.48 , s.d. 0.99). The post-hoc tests showed that these differences were significant between all levels of Input Technique ( $p<=0.012$ ) except between Mouse and Flick, Mouse and Relative, and Token and Absolute. Most striking is that both absolute conditions introduced much larger overhead to scrolling than the relative techniques and the control condition.

\subsubsection{Jumps during Absolute Scrolling}

In contrast to relative scrolling, Absolute scrolling allows participants to perform large jumps during navigation, by touching the corresponding point on the touchpad. Jumping can be a desired effect, to efficiently navigate to a target area without having to actually perform much movement on the touchpad. However, jumps can also be introduced accidentally. During the experiment, we observed two types of behavior that resulted in unintended jumps in the navigation:

Clutches. The most familiar and intuitive mode of operation for a touchpad is relative mapping. In relative mode, users can clutch by lifting the finger up, repositioning back to the middle and putting the finger down again to resume interaction. However, in our experiment we observed also accidental 

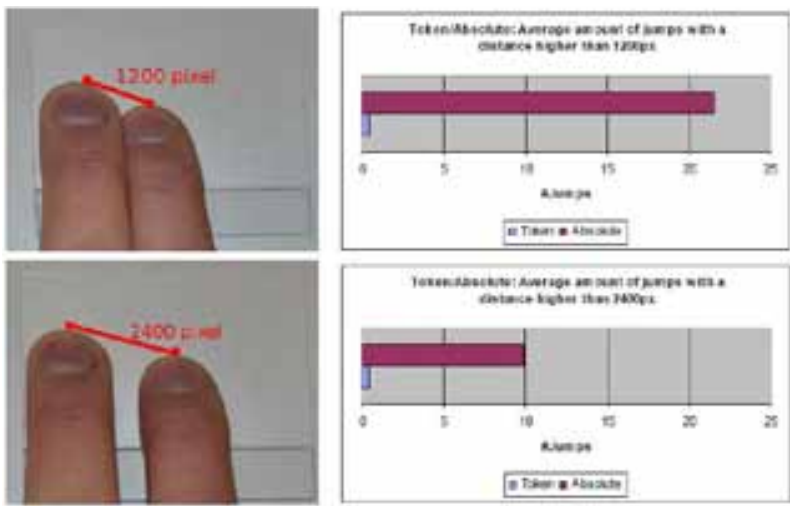

Figure 5: Recorded jumps when scrolling with Absolute, and Token.

clutching during the Absolute condition. Here, clutching does not work as intended, as bringing the finger back to a start position also causes the navigator widget to jump back.

Reacquisition offset. In the Absolute condition, some users took the finger off the touchpad in between scrolling, when they focused on mouse acquisition of target point. After clicking a target, they had to reacquire the touchpad to continue scrolling. Although aware of the absolute mapping, users did not tend to try and reacquire the touchpad at the last position, thus causing an unintended jump to new image location before they resumed scrolling to the next target.

For a quantitative analysis of jumps during scrolling, we used our activity log and applied two thresholds, 1200 pixels and 2400 pixels, corresponding to one or two finger width's on the touchpad (see Fig. 5). The mean observed across participants was 9.9 jumps above the 2400 pixel threshold (s.d. 9.5), and 21.5 above the 1200 pixel threshold (s.d. 15.5). In comparison, we hardly observed any jumps in the Token condition (mean 0.4, s.d. 0.5 for both thresholds) although this also based on absolute mapping of the touchpad. Two paired T-Tests show that there are significantly less jumps in Token than in Absolute for both 1200 pixels $(\mathrm{t}(13)=5.474, \mathrm{p}$ $<0.001)$ and 2400 pixels $(\mathrm{t}(13)=4.2, \mathrm{p}=0.001)$. This indicates that the use of a token changes the characteristics of interaction for absolute positioning on the touchpad.

\subsection{Video Analysis}

Video was recorded during all user sessions. A detailed analysis of the video material yielded two specific observations on how users interacted with the touchpad, and with the token:

Finger postures. We expected participants to interact with the touchpad using the tip of a finger. However, the video analysis showed that some participants only used a very light touch, and sometimes even only their fingernail, in which case the scrolling mechanism would not respond. On the other extreme, some participants used not only the tip, but a large part of the finger to touch the surface of the touchpad. Both of those behaviours particularly affected Absolute. In this condition, a light touch would not trigger any scrolling, prompting users to touch again and harder which then resulted in jump in the navigation. The other extreme, a touch with a large of a finger, made it difficult to provide absolute input near the edge of the touchpad, because the capacitive sensors pick up the center of gravity of the touch (i.e., a lighter touch was required in order to reach the edge of the touchpad). These two problems are specific to absolute input. With relative scrolling, a missed input due to light touch is naturally recovered by clutching until the desired movement is achieved, and large contact is unproblematic as there is no need to select input at the edge of the touchpad).

Token Interaction. The participants were expected to place their finger centrally on the coin to move it and to provide input. However, many users instead shifted the coin on the touchpad by pushing or pulling it with the finger at the edge of the coin. This interaction behaviour caused multiple problems. When the token was only lightly touched at its side for repositioning, no input was registered and the touchpad failed to track the coin position, resulting in unintended jumps. In other cases the finger position was sensed but represented the edge rather than the centre of the coin. This in turn caused problems when users tried to navigate to the edge of the input space.

\subsection{Qualitative Results}

We asked the participants to grade the scrolling techniques based on a 5-level Likert scale. A Friedmann ANOVA shows a significant effect for all items $(p<0.009)$. Wilcoxon post-hoc test show an similar result for each item: there was no significant difference between Mouse, Relative and Flick. However, generally at least one of the absolute conditions Absolute or Token was rated significantly worse compared to either Mouse, Relative or Flick ( $\mathrm{p}$ $<0.032$ for each pair-wise comparison) for the items Simplicity, Performance, Efficiency, Comfort, Like, Ease of Learning, Frustration Level, Satisfaction and Mental Demand. The participants also ranked the techniques. A Friedman ANOVA shows that there is a significant difference between the interactions $(\mathrm{Xs}(4)=19.307, p=0.001)$. Mouse (mean 4.0, s.d. 1.07) and Relative (mean 3.53, s.d. 1.55) were the preferred techniques, followed by Flick (mean 3.33, s.d. 0.90). Absolute (mean 1.87, s.d. 0.64) and Token (mean 2.67, s.d. 1.58) were least preferred.

Participants were invited to comment on each of 
the scrolling techniques. Common feedback was that Mouse is "more comfortable" and that they are "used to" this technique. Relative was mentioned to be "easy" but also "exhausting". Flick and its inertia effect received mixed feedback, with half of the participants expressing that they liked the technique, whereas the other half noted that they had difficulties with this technique; some of the participants mentioned problems with stopping the inertia effect. Token also received mixed feedback. Some of the participants noted that Token is "natural and easy", others found it "too sensitive". One participant mentioned that Token feels "awkward and unnatural" as the touchpad is not tapped directly. Absolute predominantly received negative feedback. Participants expressed that this technique was "jumpy", "difficult" and "tiring the wrist". One participant noted that Absolute is "more guessing" than precisely navigating.

Participants were also asked in which settings they would use notebooks in an $\mathrm{N}+\mathrm{M}$ configuration, and potentially take advantage of two-handed interaction. Mentioned settings varied between home and office, but generally the configuration would be preferred in a stationary setting. One participant suggested that he would use the two-handed configuration everywhere as it was not as tiring as the standard mouse interaction and prevented repetitive strain injury.

\section{DISCUSSION}

Relative scrolling. Our experiments show that participants were able to instantly use both relative scrolling techniques and perform as well as in the familiar control condition Mouse. However, the experiment did not show a significant gain in performance by using both hands in our study task. This was not unexpected, as users are very familiar with using the mouse for scrolling (as confirmed by our qualitative results), while navigating with the non-dominant hand using the touchpad is novel. The measured learning effect suggests that in particular Relative has potential to outperform onehanded scrolling with more training. The addition of flicking and inertia did not significantly influence the performance and the user's perception of scrolling. However, the act of flicking - while saving movement over longer distances - has been shown to introduce significantly more overhead in terms of the distances that were navigated, compared to relative navigation without inertia.

Absolute scrolling. Both absolute scrolling techniques performed significantly worse than the other techniques in all aspects. Absolute and Token were less efficient, produced more overshoots, introduced more navigational overhead and was rated consistently worse than the other techniques. However, for both absolute conditions we observed a very steep learning curve, which indicates that users had not reached their maximum performance during the relatively short duration of the experiment. As indirect absolute input is not a common interaction in practice, it can be assumed that users were less familiar with such an interaction than with relative input for scrolling. This would explain why absolute scrolling would be initially less efficient, more error-prone and as a consequence rated worse by participants. The recorded learning curves for both Absolute and Token were steeper than for relative scrolling and indicate that both techniques have significant potential to improve over longer periods of usage.

The use of a token for absolute navigation had a strong effect. Our study shows that by merely adding a coin to the touchpad's surface a different style of interaction is encouraged. The token acts as a tangible indicator for the current position of the navigation, making it easy and intuitive to re-acquire the current absolute position on the touchpad in case the finger is removed from the touchpad in between interactions. The token also seemed inherently eliminate the reflex of clutching which we observed during absolute scrolling with direct finger input. Consequently, the use of a token is effective for avoiding unintended jumps during absolute scrolling.

It is important to note that absolute scrolling does not scale with the size of the overall virtual canvas in the same way as the other techniques do. In our experiment, the size of the touchpad (65 $\times 40 \mathrm{~mm}$ ) and the available input resolution were mapped onto a canvas size was $6000 \times 6000$ pixels. Increasing the canvas size would further emphasize accidental jumps and overshoots, while decreasing the canvas size might reduce and even eliminate problems that were measured in this study. Consequently, the data presented in this study is limited to the specific parameters that applied.

Alternative ways of scrolling. The study assumed that a navigator widget as described is representative of the state of the art for 2D scrolling. This is backed up by the provision of a navigator widget for $2 \mathrm{D}$ scrolling in many software applications, such as Photoshop, CorelDraw or Gimp. However there are other ways of scrolling, in particular scroll bars and dedicated keys on the keyboard. Scroll bars require two separate interactions with the horizontal and vertical scrollbar, and have to be considered less efficient and expressive than navigator widgets. Keyboard scrolling is similar to our technique in that it can be performed with the non-dominant hand, but it involves an inherent trade-off between accuracy and speed (i.e., depending on how many pixels are 
scrolled per key press, navigation is either accurate or fast). Keyboard scrolling is also less expressive, as it is limited to scrolling in eight directions. In contrast, the techniques we presented give the user explicit control both over the velocity and the direction of scrolling.

ID scrolling. All of the presented 2D scrolling techniques can be projected onto their ID equivalent, by disregarding one of the dimensions. For instance, flick could be used to vertically navigate large documents in programs such as word processors. Similarly, relative scrolling techniques could be used horizontally, for instance to scroll time-lines (e.g., common for video editing). While this broadens the application range of the introduced scrolling techniques, it is important to note that the results of the empirical study are specific to 2D scrolling and cannot be directly transferred to ID scrolling.

Task and practical impact. The study task was designed to be representative of typical workflows that involve a combination of scrolling across larger output spaces and manipulation of detail in such spaces, such as navigating and editing maps, manipulating vector graphics, editing images, and playing strategy games. We generally consider our results applicable to such workflows, but it is important to note that we used a "compressed" workflow for our study, in which a single click is used to simulate interaction in between scrolling. While this does not influence the act of scrolling itself - which was our focus - it produces a shorter overall tasks completion time in which the effect of the navigation techniques might be over-pronounced. Whether our techniques can lead to actual performance gains in practice is dependent on the task. There are workflows, such as retouching or strategy games, that are very close to our artificial task, and in which touchpad scrolling can potentially have a significant impact on the user's performance. However, in other applications, such as editing the splines of a vector graphic, significantly more interaction takes place in between scrolling, which means that scrolling weights less for overall performance and a two-handed approach would have much more limited impact.

Finally, it is important to note that independent from quantitative factors, the introduced techniques also add qualitative benefits to scrolling, which have not been subject of this study. Users can distribute certain tasks across two hands, which has been shown to be beneficial in experimental research as well as in professional practice, for example in CAD. Moreover, touchpad scrolling enables the user to perform actions that were not possible before, such as scrolling while performing a drag\&drop operation. Consequently, it is an interesting question for further research how two-handed interaction with touchpad and mouse affects the expressiveness of user input.

\section{CONCLUSION}

In this paper we have shown that a common computer configuration, a notebook with external mouse $(\mathrm{N}+\mathrm{M})$, can provide support for two-handed interaction "out-of-the-box", without requirement for additional hardware. This in itself is significant, as it suggest a route to wider adoption of two-handed input.

Specifically, we have proposed to use the notebook touch-pad as a dedicated device for 2D scrolling with the non-dominant hand, while the external mouse is used for primary pointing with the dominant hand. In this context, we have shown that once decoupled from pointer input, the touchpad affords a variety of novel scrolling techniques with different interaction characteristics, including flicking with inertia, and absolute input via a token.

Our evaluation showed that users were immediately as efficient with relative scrolling techniques on the touchpad as with familiar mouse-based scrolling, but significantly less efficient with techniques that applied an absolute mapping. The study did not show an instant performance gain resulting from two-handed input, but the observed learning curves indicate a potential to outperform one-handed scrolling after more training. This prompts future work to investigate learning and adoption of the proposed techniques in a longitudinal study.

\section{ACKNOWLEDGEMENTS}

This research was conducted with support of the INTERMEDIA Network of Excellence (European Commission contract NoE 038419).

\section{REFERENCES}

[1] Spacepilot.3dconnexion. http:// www.3dconnexion.com/3dmouse/spacepilot.php.

[2] Synaptics touchpad interfacing guide: http://www.synaptics.com/sites/default/files/511000275-01ra.pdf.

[3] Balakrishnan, R. and Hinckley, K. (1999) The role of kinesthetic reference frames in two-handed input performance. In Proc. UIST '99, pages 171-178, New York, NY, USA. ACM.

[4] Balakrishnan, R. and P. Patel. (1998) The padmouse: facilitating selection and spatial positioning for the non-dominant hand. In Proc. $\mathrm{CHI}$ '98, pages 9-16, New York, NY, USA. ACM Press/ 
Addison-Wesley Publishing Co.

[5] Buxton, W., Hill, R. and Rowley, P. (1985) Issues and techniques in touch-sensitive tablet input. In Proc. SIGGRAPH '85, pages 215-224, New York, NY, USA. ACM.

[6] Buxton, W. and Myers, B. (1986) A study in twohanded input. SIGCHI Bulletin, 17(4):321-326.

[7] Fallot-Burghardt, W., Fjeld, M., Speirs, C., Ziegenspeck, S., Krueger, H., and Láubli, T. (2006) Touch\&type: a novel pointing device for notebook computers. In Proc. NordiCHI '06, pages 465-468, New York, NY, USA. ACM.

[8] Guiard, Y. (1987) Asymmetric division of labor in human skilled bimanual action: The kinematic chain as a model. Journal of Motor Behavior, 19(4):486517.

[9] Harris, C. and Straker, L. (2000) Survey of physical ergonomics issues associated with school children's use of laptop computers. International Journal of Industrial Ergonomics, 26(3):337 - 346.

[10] Hinckley, K., Czerwinski, M. and Sinclair, M. (1998) Interaction and modeling techniques for desktop two-handed input. In Proc. UIST '98, pages 49-58, New York, NY, USA. ACM.

[11] Kabbash, P., Buxton, W. and Sellen, A. (1994) Two-handed input in a compound task. In Proc. $\mathrm{CHI}$ '94, pages 417-423, New York, NY, USA. ACM.

[12] Kabbash, P., MacKenzie, I. S. and Buxton, W. (1993) Human performance using computer input devices in the preferred and non-preferred hands. In Proc. $\mathrm{CHI}$ '93, pages 474-481, New York, NY, USA. ACM.

[13] Kurtenbach, G., Fitzmaurice, G., Baudel, T. and Buxton, B. (1997) The design of a GUI paradigm based on tablets, two-hands, and transparency. In Proc. CHI '97, pages 35-42, New York, NY, USA. ACM.

[14] Odell, D. L., Davis, R. C., Smith, A. and Wright, P. K. (2004) Toolglasses, marking menus, and hotkeys: a comparison of one and two-handed command selection techniques. In GI '04: Proceedings of Graphics Interface 2004, pages 17-24, School of Computer Science, University of Waterloo, Waterloo, Ontario, Canada. Canadian Human-Computer Communications Society.

[15] Rekimoto, J. (2002) Smartskin: an infrastructure for freehand manipulation on interactive surfaces. In Proc. CHI '02, pages 113-120, New York, NY, USA. ACM.

[16] Rekimoto, J. (2003) Thumbsense: automatic input mode sensing for touchpad-based interactions. In Proc. CHI '03, pages 852-853, New York, NY, USA. ACM.

[17] Sawin, D. A., Stewart, A. M. and Calcaterra, J. A. (2002) Pointing stick versus touch pad: Working together. Human Factors and Ergonomics Society Annual Meeting Proceedings, 46:607-611(5).

[18] Sommerich, C. M. (2002) A survey of desktop and notebook computer use by professionals.
Human Factors and Ergonomics Society Annual Meeting Proceedings, 46:1124-1128(5).

[19] Woodruff, A., Anderson, K., Mainwaring, S. D. and Aipperspach, R. (2006) Portable, but not mobile: A study of wireless laptops in the home. Technical report EECS-2006-88.

[20] Zeleznik, R. C., Forsberg, A. S. and Strauss, P. S. (2997) Two pointer input for 3D interaction. In SI3D '97: Proceedings of the 1997 symposium on Interactive 3D graphics, pages 115-120, New York, NY, USA. ACM. 